\title{
Estilo de gerencia de tecnología en unidades internacionales de producción radiofónica infantil
}

\author{
Petit Torres, Elsa Emilia* \\ * Universidad del Zulia. Facultad Experimental de Ciencias. Departamento de Ciencias \\ Humanas. Unidad Académica Estudios del Desarrollo. E-mail: eept@latinmail.com
}

\section{Resumen}

En el presente artículo se identifican las principales características del estilo de gerencia de tecnología en unidades internacionales de producción radiofónica infantil. La estrategia de investigación es cualitativa basada en el método estudio de casos, en dos fases: exploratoria y descriptiva, conforme al proceso dialéctico propuesto por Martínez (1996). Los casos seleccionados fueron: La Chacamota de México, Colorín Color Radio de Colombia, Radio Lollipop de Inglaterra y Radio Tic-Tac de Estados Unidos. El estilo de gerencia de tecnología en unidades internacionales de producción radiofónica infantil se caracteriza por: combinar capacidades internas y externas, implementar nuevas tecnologías, diversificar la cartera de productos, gestionar información y recursos y transmitir y vender el servicio a través de internet, gestionar redes de información y acción. En conclusión, la gerencia de tecnología en la producción internacional de radio para niños se orienta sistemáticamente a la innovación en producto, por medio de la estrategia para desarrollar competitividad basada en la integración de las capacidades dinámicas de la organización. Esta investigación constituye una base de datos útil para futuras investigaciones de mayor complejidad y profundidad referidas a la gerencia de tecnología en la producción radiofónica infantil de cualquier país en el mundo.

Palabras clave: Gerencia de tecnología, producción radiofónica infantil, innovación en producto, competitividad.

\section{Technology Management Style in International Production Units in the Child Radiophonic Sector}

\begin{abstract}
This article identifies the principal characteristics of technology management style in international units of child radiophonic production. The research strategy was qualitative based on a two-phase case study (exploratory and descriptive) according to the dialectic
\end{abstract}


process proposed by Martinez (1996). The cases selected were: La Chacamota from México, Colorín Color Radio from Colombia, Radio Lollipop from England, and Radio TicTac from the United States. The technological management style utilized in international unit child radiophonic production are characterized by: combinations of external and internal capacities, new technology implementation, diversification in the product line, information and resource management, and transmission and sales service by internet, through information and action webs. In conclusion, technology generation in international production of radios for children is systematically oriented towards product innovation, by means of strategies to develop competitiveness based on integration of dynamic organizational capacities. This research constitutes a data base for more complex and profound future research referring to technology management in child radiophonic production in any country in the world.

Recibido: 03-02-16. Aceptado: 04-04-23

Key words: Technology management, child radiophnic production, product innovation, competition.

\section{I ntroducción}

Por medio de la investigación adelantado se identifican las principales características del estilo de gerencia de tecnología en unidades internacionales de producción radiofónica infantil (UIPRI), con el propósito de establecer una base de datos útil para futuras investigaciones de mayor complejidad y profundidad referidas a la gerencia de tecnología en la producción radiofónica infantil de cualquier país en el mundo.

De la revisión bibliográfica efectuada para el estudio, de acuerdo con los recursos de información disponibles, no se desprendió evidencia alguna sobre la existencia de investigaciones referidas al tema de la gerencia de tecnología en unidades internacionales de producción de radio para niños.

La búsqueda bibliográfica sobre el tema de gerencia de tecnología indicó la existencia de estudios referidos a la industria manufacturera, lo cual no se adaptaba a la naturaleza de las empresas radiales, a sus procesos y productos. Sin embargo, la teoría de innovación refería modelos generales para el caso de innovación en producto e innovación en proceso, respectivamente. La consideración de tales modelos de innovación, reorientó el desarrollo del marco teórico del estudio para obtener resultados importantes y útiles, a los productores de radio infantil, para promover la gerencia de tecnología dentro de sus 
empresas, como una estrategia para estimular la producción competitiva e innovadora.

Dada la carencia de estudios en el área de la gerencia de tecnología en unidades de producción radiofónica $y$, en consecuencia, a las dificultades para establecer un marco conceptual y analítico apropiado, para analizar estilos de gerencia de tecnología en casos de productoras de radio infantil en Venezuela, se inició un proceso de búsqueda de información sobre experiencias "competitivas" de producción radial infantil, es decir, aquellas experiencias internacionales con éxito comercial, para identificar cómo se manejan las decisiones tecnológicas en tales unidades productoras.

El contenido de este artículo genera un nuevo planteamiento teórico: debido a que se considera a la tecnología, no sólo como dispositivos físicos: equipos y máquinas, sino como todo aquél conocimiento tácito, que aunado al medio técnico de producción, es útil para el fortalecimiento de la industria de la radio, concretamente en el ámbito de las productoras del género infantil.

Este trabajo es un primer intento de acercamiento de la teoría sobre gerencia de tecnología, al contexto de la producción en empresa radial. Los resultados de este estudio pueden desencadenar una serie de investigaciones referidas a la generación de estrategias, modelos y propuestas teóricas de gestión tecnológica para crear nuevos productos así como procesos, que contribuyan a desarrollar, de manera competitiva, la producción radiofónica para ni ños.

Con los resultados de este estudio se activa la producción científica en materia de radiodifusión infantil y gerencia de tecnología, además se valoriza a la gerencia de tecnología como fundamento de éxito, crecimiento económico de la empresa radial tanto en los de tipo general así como de las pequeñas y medianas empresas (PYMES) ligadas a la producción radiofónica dirigida a la población infantil.

Con el contenido de este artículo se busca difundir las principales características del estilo de gerencia de tecnología de las UIPRI como un aporte valioso a los nuevos emprendedores, interesados en integrase al trabajo de la producción radial dedicada a los niños en cualquier emisora o productora de radio a nivel internacional, donde se considere la producción del género. Los resultados presentados sirven de base para diseñar estrategias que contengan un conjunto de nuevas prácticas en materia tecnológica y dentro de la unidad de producción radial infantil para incorporar atributos tanto en procesos como en productos, contribuyendo a elevar sus niveles de competitividad. 


\section{Metodología}

Para alcanzar el objetivo referido al estudio de las UIPRI, se seleccionaron cuatro casos de distintos países. Uno de los casos fue "LA CHACAMOTA" de México (LCH). El resto de las unidades productivas seleccionadas para el análisis fueron: COLORÍN ColorRadio de Colombia (CCR), RADIO TIC- TAC de Estados Unidos (RTT), y RADIO LOLLIPOP de Inglaterra, Nueva Zelanda y Estados Unidos (RL).

Para realizar el estudio sobre el estilo de gerencia de tecnología en las UIPRI, se adoptó una estrategia de investigación cualitativa basada en el método de estudios de casos. La estrategia se cumplió en dos fases; fase I exploratoria y fase II descriptiva.

Esta estrategia fue seleccionada como la más apropiada en razón del carácter exploratorio de la investigación, donde el tema a estudiar, la gerencia de tecnología en unidades de producción radiofónica, en general, y en particular, en el caso de programas para niños, no ha sido objeto de previas investigaciones. En segundo lugar, esta investigación explora procesos sociales, entendidos como una estructura de significados, y no como la frecuencia estadística de la ocurrencia de un fenómeno (Martínez, 1996). El proceso seguido para el desarrollo del estudio es descrito a continuación.

La primera fase del estudio es exploratoria. Para ella se inició la búsqueda de información sobre experiencias de producción radiofónica para niños en el contexto internacional, considerando ciertos criterios de selección explicados más adelante. El primer caso internacional fue seleccionado por la relación profesional que la investigadora tiene con la productora "LA CHACAMOTA" de México y porque el programa cumple con la característica de ser comercialmente exitoso.

Para completar el conjunto de casos seleccionados como corpus de análisis se inició un proceso de búsqueda de información vía internet para conseguir datos sobre empresas de producción radial infantil, se consiguió una infinidad de documentos que describían proyectos, programas y empresas ligadas a la producción radial infantil. Del total de información obtenida se seleccionó el resto de los casos, conforme a los siguientes criterios: 1) disponibilidad de información en la WEB, 2) cantidad y calidad de información suministrada en la WEB, 3) los tres casos cumplían con la característica de tener permanencia (es decir, no eran experiencias transitorias o esporádicas) en programación y 4) los tres casos seleccionados en la WEB cumplían con la característica de ser exitosos desde el punto de vista comercial (el criterio de selección será explicado más adelante). Esta información fue impresa en papel para su posterior estudio. 
La recolección de información para el caso de la unidad producción "LA CHACAMOTA", se consiguió a través de un cuestionario enviado mediante el sistema de correo postal, a finales del año 1999, y devuelto a principios del año 2000. En el resto de los casos el procedimiento se hizo a través de la World Wide Web (WWW), lográndose la recolección de datos mediante la Página WEB correspondiente.

El análisis de la información fue un proceso que consistió en la construcción de una caracterización que explicara lo más precisa y claramente posible el objeto estudiado. Para lograr la descripción de la estructura de casos de estudio, se desarrolló un proceso "dialéctico" en la mente de la investigadora, un acto por descubrir la estructura (Martínez, 1996). Este esfuerzo por comprender la realidad de cada caso analizado se apoyó en los conocimientos acumulados durante la experiencia de formación académica de la investigadora en las escolaridades realizadas: Comunicación e Industrias Audiovisuales en La Universidad Internacional de Andalucía en Huelva (España) y Planificación y Gerencia de Ciencia y Tecnología en La Universidad del Zulia en Maracaibo (Venezuela).

Con base en los conocimientos anteriores se aplicó análisis de contenido a los datos recolectados de cada caso, surgieron categorías y subcategorías que se identificaron como los procesos más significativos de la gerencia de producción en cada caso analizado. La identificación de los procesos más significativos se hizo mediante la construcción de un cuadro, donde se cuantificó la frecuencia de observaciones hechas, por cada categoría y caso de estudio.

Como resultado del proceso anterior, se construyó el marco conceptual y analítico, mediante la abstracción donde, primero, se identificaron los aspectos gerenciales envueltos en la producción de programas radiales y segundo, se construyeron categorías conceptuales referidas a la gerencia de tecnología. Esta dinámica permitió reenfocar la revisión bibliográfica hacia las áreas más relevantes para el tema de investigación y de este modo se construyó el marco conceptual y analítico requerido.

Para la fase descriptiva de la investigación se analizó la información sobre los casos mediante la aplicación de la técnica de análisis de contenido, los resultados se interpretaron a la luz del marco conceptual analítico.

Con la aplicación del análisis del contenido surgieron nuevas categorías y subcategorías que se identifican como los procesos más significativos de la gerencia del producto en cada caso analizado. Finalmente se genera la síntesis descriptiva que contiene los 
resultados de esta investigación. Estos resultados no pueden ser generalizados más allá de los casos de estudio. Se requiere replicar los resultados en un mayor número de casos. Esta investigación es referida únicamente al sector radiofónico infantil de países específicos. Sus resultados no pueden ser generalizados a otras áreas de la producción radial sin antes realizar las adaptaciones necesarias a aplicaciones particulares.

\section{Gerencia y Gerencia de Tecnología}

Dentro del marco de la economía de la tercera Ola프, un aspecto fundamental para el desarrollo de la gerencia viene a ser la consideración de la tecnología, el know how, la forma de producir como un factor clave para desplegar la competitividad en las empresas. Por lo tanto, se entenderá como gerencia de tecnología, a las implicaciones del cambio tecnológico en la estrategia de la empresa (Smail Ait-El Hadj, 1990). La gerencia de tecnología consiste en el manejo de las decisiones tecnológicas por parte de la empresa (Ávalos, 1994). La gerencia de tecnología es parte de la estrategia corporativa hacia una posición competitiva de la firma.

La tecnología no sólo abarca dispositivos físicos y equipos (tecnología dura); la tecnología debe entenderse también como el medio para llevar a cabo una tarea, incluye lo que es necesario para convertir recursos en productos o servicios, el conocimiento y los recursos (tecnología blanda) para lograr un objetivo; es el cuerpo de conocimientos científico y de ingeniería que puede aplicarse en el diseño de productos y / o procesos o en la búsqueda de un nuevo conocimiento ( "Gus" Gaynor, 1999).

La gestión de tecnología puede describirse como el proceso de integrar los recursos y la infraestructura de la unidad de negocios en el logro de sus propósitos, sus objetivos, sus estrategias y sus operaciones definidos ("Gus" Gaynor, 1999). Así pues, la gestión de tecnología se constituye en la fuerza motriz de la mayor parte de las organizaciones, en particular, de aquellas que les preocupa el futuro.

Gerencia es sinónimo de dirección o management, un proceso integrador en el que específicas personas gestionan y canalizan el esfuerzo productivo de una empresa $u$ organización $\underline{2}$ o parte de ella hacia el cumplimiento de determinadas metas y objetivos (Rojas, 1994). El término con el que en inglés se traduce "gerencia" es "management". Asimismo para designar gestión", se utilizan diversos sentidos que tiene "gestión"; estos son: “promotión”, “negociatión”, “actión”, “proceding” y también “management" (Barreto, 2000). 
Al iniciar ésta reflexión teórica, se planteó la gerencia de tecnología, como una técnica moderna para orientar la estrategia corporativa hacia una posición competitiva. Al respecto, el Director de Promoción de Inversiones y Tecnología de la Organización de las Naciones Unidas para el Desarrollo Industrial (ONUDI), Fernando Machado (1996) , afirma que la innovación tecnológica juega un papel absolutamente fundamental en el establecimiento de la competitividad sostenible a nivel de la empresa y que mediante una gestión inteligente, la misma se constituye en el vector integrador de las siguientes posibilidades estratégicas de diferenciación: 1) como estimulante de alianzas estratégicas internacionales, de la participación de redes y grupos de empresas, 2) como el mecanismo por excelencia para posibilitar a la empresa a realizar la rearquitectura de su negocio y 3) como instrumento a través del cual la empresa desarrolla nuevos negocios.

En referencia al tema de la competitividad en la empresa, el autor Machado (1996) explica, que si en la empresa no se manejan los cambios tecnológicos de una manera integrada con los cambio técnicos, organizativos, estratégicos financieros y gerenciales, muy probablemente el proceso de innovación tecnológica fracasará, además acota en su postura, que "la gestión tecnológica es el elemento integrador de dichos cambios".

Los autores Tidd, Bessant y Pavitt (1997), también consideran que existe una vinculación entre la estrategia tecnológica de la firma y la posición competitiva y de mercado. Además, reflexionan sobre el importante y doble rol que desempeña la innovación, como una fuente de incertidumbre; como cambio en el ambiente y a la vez, como recurso dentro de la firma para desarrollar competitividad.

La innovación debe ser considerada por la gerencia de tecnología como recurso dentro de la firma para desarrollar competitividad. Al respecto, Tidd, Bessant y Pavitt (1997), desarrollaron un marco de análisis para definir e implementar la estrategia innovativa de la corporación, logrando conclusiones importantes sobre cuatro enfoques: RACIONALISTA, "SWOT", INCREMENTALISTA Y CAPACIDADES DINÁMICAS DE LA EMPRESA. Estas conclusiones fueron las siguientes 1) los enfoques: racionalista, "SWOT" e incrementalista, subestiman el poder del cambio tecnológico para trastocar mercados establecidos y condiciones de competitividad existente, y sobrestiman la influencia que el gerente, o que los gerentes realmente tienen sobre la selección de una estrategia tecnológica, 2) el marco más apropiado para el diseño de las estrategias corporativas es el enfoque de las capacidades dinámicas propuesto por David Teece y Gary Pisano (1994).

Tidd, Bessant y Pavitt (1997) aseguran que la estrategia corporativa que no reconoce la complejidad del presente y las incertidumbres asociadas al cambio y el futuro, ciertamente 
serán rígidas, probablemente estarán equivocadas y potencialmente serán desastrosas si son completamente implementadas.

\section{Las capacidades dinámicas de la firma}

Tidd, Bessant y Pavitt (1997) concluyen en su estudio, que el marco más apropiado para el diseño de las estrategias corporativas es el enfoque de las capacidades dinámicas propuesto por Teece y Pisano (1994). Este enfoque subraya la importancia del cambio dinámico y el aprendizaje corporativo, considera el carácter cambiante del ambiente y hace énfasis en el importante papel de la dirección estratégica adaptando, reconfigurando e integrando apropiadamente habilidades organizacionales internas y externas, recursos y competencias funcionales hacia un entorno cambiante.

Según Teece y Pisano (1994) una capacidad para que sea estratégica, debe ajustarse a la necesidad del usuario, para que el producto sea único y difícil de reproducir. Las dimensiones estratégicas de la empresa son su proceso gerencial y organizacional, su posición y caminos disponibles. Por procesos gerenciales se entiende, la manera de hacer las cosas en la empresa; sus rutinas, modelos de práctica actual y lo que se podría estar aprendiendo. Por posición, se entiende, la dotación actual de la tecnología y la propiedad intelectual, así como su base del cliente y relaciones con proveedores. Las alternativas estratégicas disponibles a la empresa y el atractivo de oportunidades que quedan por delante es lo que Teece y Pisano, denotan como el camino. En śntesis, este enfoque considera la combinación de tres estrategias básicas: de mercado, tecnológica de la firma y organizacional.

Dentro de este marco conceptual y analítico también es importante destacar que el producto generado por la empresa de producción radiofónica posee características muy particulares, es único e intangible. Intangible porque es invisible e inmaterial, diferente de cualquier producto manufacturado, y único, porque implica un carácter no estandarizado en su fase de diseño, este aspecto lleva consigo que el producto radiofónico se someta a cambios constantemente en su fase más temprana de producción: en el diseño.

\section{El modelo del proceso de innovación y el ciclo de vida del producto}

En 1978 Abernathy y Utterback explican el modelo del proceso de innovación asociado al ciclo de vida del producto. Estos autores plantean dos formas particulares de innovación en unidades productivas: 1) el patrón de las grandes unidades de producción que generan altos volúmenes de un producto standard, donde la innovación es incremental y con efecto 
gradual y acumulativo en la productividad; orientada a mejorar el desempeño de sistemas de producción existentes mediante mejoras en los métodos de fabricación, la distribución y el mercadeo. 2) el patrón de innovación radical en pequeñas empresas donde estas se asocian a la acción de identificar una necesidad emergente (innovación en producto) o con una nueva manera de satisfacer una necesidad existente (innovación en proceso). La innovación industrial es, entonces, un proceso dinámico que evoluciona dentro de estos dos patrones extremos correspondientes a los tipos básicos de innovaciones: radicales e incrementales, a medida que las organizaciones crecen y maduran. Este modelo tiene implicaciones para la gerencia de innovación tecnológica, al proponer que el cambio en la estrategia corporativa de la unidad de innovación industrial ocurre en la medida que, la unidad madura de una posición pionera a una posición de productor de gran escala (Añez, 1995).

\section{La gestión estratégica y operativa de la tecnología}

Los estudios adelantados por Tidd, Bessant y Pavitt (1997), concuerdan con algunas ideas aportadas por Smail-Ait-El Hadj (1990), quien ha estudiado la gestión de tecnología desde dos perspectivas convergentes: LA GESTIÓN OPERATIVA DE LA TECNOLOGÍA, cuyos ámbitos de gestión son: productos, procesos y conocimientos o información, éstos tres ámbitos se relacionan respectivamente con los ámbitos de LA GESTI ÓN ESTRATÉGICA DE LA TECNOLOGÍA: posicionamientos o capacidades, maniobras, sinergias y alianzas, y vigilia, previsión, análisis y competencia.

Ait-El Hadj (1990), es uno de los autores que considera la gestión de tecnología, como un instrumento de la empresa para responder a la innovación, su idea es que la gerencia esté pendiente del entorno y de la estructura interna, para aprovechar todas las ocasiones para mejorar los productos y los procesos de la empresa y para hacerla más competitiva.

En las empresas la tecnología puede percibirse como producto, proceso o forma de organización. Para cada tipo de tecnología se ha establecido un modo de gerencia: 1) Para la tecnología en producto; la gerencia de productos, 2) Para la tecnología de procesos, la gerencia de procesos y 3) Para la configuración de la organización se ha establecido la gestión organizacional. Asimismo, Ait- EL Hadj (1990), ha definido lugares de aplicaciones del proceso de innovación, entre los cuales señala: a) El de producto o innovación externa, y b) El de proceso o innovación interna.

La primera según Ait- El Hadj (1990), permite a la empresa ofertar mejores productos que los existentes en el mercado, ya que ofrecen más funcionalidades, o cumplen 
funciones de manera más eficaz, siendo más ligeros, menos voluminosos, más sencillos, entre otros. También puede tratarse de productos totalmente nuevos. En el caso que nos ocupa, el producto radiofónico puede considerarse un tipo de producto único, el cual se recrea en cada acto productivo.

Los mecanismos empleados desde la empresa para innovar en producto tienen relación con la actividad de gestionar la información como clave para orientar la producción a la satisfacción del cliente. Al respecto, Ait- El-Hadj (1990) menciona algunos mecanismos para innovar en producto de modo sistémico: 1) vigilia: expresa la inquietud por parte de la empresa de asegurar permanentemente una vigilancia con relación a un universo cambiante. Es una actividad organizada de la empresa, manifiesta la intención de estructurar, de finalizar y de esta manera convertir en perenne la búsqueda de información en el exterior, 2) redes: para la empresa son medios de disponer del mayor número de vínculos, para cubrir el campo de la información y acción.

Ait-El Hadj (1990) plantea además una taxonomía muy completa sobre los tipos de redes descrita a continuación:

- Red para la acción: Esta red puede tener múltiples formas sobre todo si se incluye el desarrollo internacional y la acción comercial. El desarrollo de este tipo de red se apoya en dos características de la industria contemporánea: 1) la complejidad tecnológica de la actividad industrial y particularmente la dispersión de los conocimientos de las tecnologías que van apareciendo, obligan a recurrir a numerosas empresas para poder realizar nuevos objetos técnicos, y 2) el paso de una concepción de la producción en términos de integración física a una concepción en términos de coherencia de las decisiones, de integración de la información y del control de la calidad y satisfacción del cliente.

- Red para la información: Este tipo de red nace de la superabundancia de información, de la dispersión de sus fuentes y de una desviación cualitativa entre la información espontánea ofrecida y las necesidades efectivas y específicas de sus solicitantes.

- Red para la investigación: Deriva de la red para la información, teniendo la investigación una dimensión de producción sistemática de información, pero también tiene una dimensión de proyecto y de acción que la distingue metodológicamente de la red para la información.

- Red de asociados equivalentes: Esta red permite la relación de empresas del mismo nivel económico y con el mismo estatuto. Cuando la iniciativa de la operación es por naturaleza colectiva podemos imaginar tres tipos de redes: 
- Red convergente: surge de reagrupar varios asociados para realizar una operación. La mayoría de las veces, se trata de la reagrupación de las pequeñas y medianas empresas (PYMES) para realizar un desarrollo común o un nuevo producto, o para mejorar o adquirir un nuevo equipamiento.

- Red en rastrillo o en estrella: destinada a asegurar una máxima cobertura del entorno, donde cada asociado decide poner en común los elementos de que dispone con los demás asociados de la red.

- Red de doble malla: esta forma más compleja contribuye a relacionar dos redes organizadas.

- Red de conocimiento: Esta es una red de alianza influenciada por el factor tecnológico para realizar productos o para tener acceso a los mercados.

- Red de sinergia: tiene por objetivo optimizar el acceso a la información pertinente para la empresa. Esta red es un medio para la empresa de disponer de mayor número de vínculos para analizar y comprender las evoluciones en curso y poner en común competencias y medios complementarios.

Los mecanismos usados desde la empresa para la gestión de la innovación tecnológica en proceso o innovación interna según Ait- El-Hadj (1990), están destinados a mejorar sistemáticamente las capacidades de rapidez, agilidad y calidad de las funciones de producción. Uno de estos mecanismos es la automatización; la utilización de software o nuevas tecnologías para elevar los niveles de productividad, flexibilidad y calidad de los procesos productivos.

\section{La nueva concepción de producción radial}

El concepto convencional de producción radial abarca la comunicación entre los procesos: pre-producción, producción y post-producción. El nuevo concepto suma al modelo anterior, el proceso de mercadear el producto. Esta mejora concebida por Bethencourt (1998) está basada en la utilización de la tecnología mercadeo como mecanismo para satisfacer las necesidades de los clientes y de la audiencia; con una nueva interpretación que integra las tecnologías de información a la gerencia del producto radiofónico.

El Diagrama 1 permite visualizar la diferencia entre el concepto de producción radial convencional, y el nuevo concepto de producción radial que maneja Bethencourt (1998). 
Diagrama 1

Concepto de producción radial convencional

vs. la nueva concepción de producción radial

Flujo material

Flujo de información

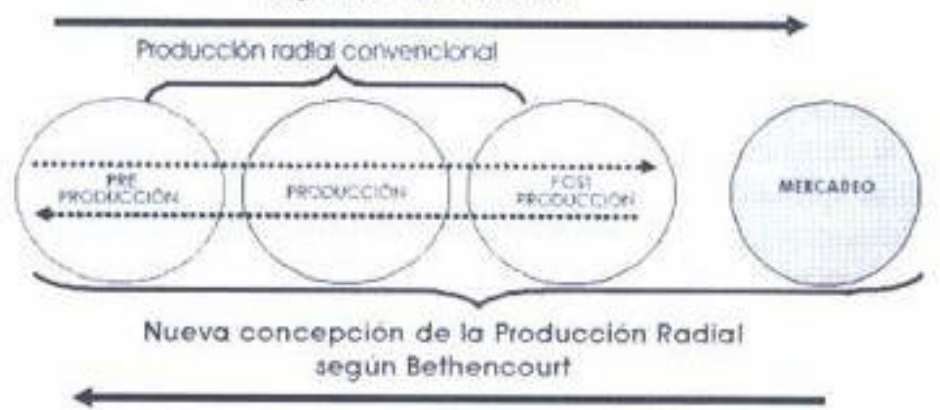

Feed Back de intormación

Fuente: Elaboracion Propia

El nuevo concepto de producción radial planteado por Bethencourt, aumenta las implicaciones de la gerencia del producto en la empresa. Los procesos de gerenciar la tecnología del producto radiofónico de acuerdo con el nuevo concepto de producción radial, permiten comprender la dinámica de retroalimentación entre la fase de mercadeo con el resto de las fases del proceso de producción radiofónica (diseño, realización, edición y transmisión) además, se percibe el proceso de mercadeo del producto como un factor clave dentro de la estrategia corporativa para innovar en producto.

\section{Resultados}

Las principales características de la gerencia de tecnología en las unidades internacionales de producción radiofónica infantil, obtenidas del estudio efectuado son:

- “COLORÍN ColorRADIO"(CCR): es una emisora de radio que produce casi 40 espacios diferentes dedicados a niños y adolescentes, transmitidos todos los días de la semana. CCR está ubicada en la ciudad de Bogotá-Colombia, desde el 11 de mayo de 1992. A partir del año 1996 se puede sintonizar en el dial 1310 AM. Para el desarrollo de sus productos la emisora cuenta con una unidad de producción, donde se integran todos los medios y recursos para realizar sus programas de radio. CCR se ha escuchado vía satélite en Quito, por el dial 1140 AM y con el programa CCR Internacional; esta emisora de radio infantil ha tenido presencia en las estaciones WSUA, 1260 AM, de Caracol Miami, U.S.A., y Radio Barrancabermeja y Caracol Armenia, en Colombia. Actualmente, transmite detalles de su programación a través del internet, esto puede evidenciarse al pulsar el hipervínculo "escúchanos" situado en su página web: http://www.colorincolorradio.com/quees.asp 
El estilo de gerenciar la tecnología en esta unidad de producción se orienta hacia la innovación en producto, característica que a su vez se traduce en mejoras del proceso productivo y considera el carácter cambiante del ambiente; al implementar nuevas tecnologías para apoyar procesos de automatización, hace énfasis en integrar apropiadamente habilidades organizacionales y los recursos internos y externos a ella. Los miembros del equipo productor CCR se integran a través de la combinación de experiencias, técnicas y conocimientos profesionales. Estos miembros logran integrarse con asesores externos para generar el producto deseado. Este equipo de asesores es lo que Ait-EL Hadj (1990), denomina red de conocimiento.

Las cualidades del personal encargado de configurar la idea de cada producto o emisión radiofónica convierten a la unidad de producción CCR en una pieza fundamental de su base tecnológica, que al combinar capacidades, con los insumos obtenidos de la red de información, se convierte en una capacidad organizacional y en un mecanismo para lograr sostener las innovaciones en producto a lo largo del tiempo. Las capacidades estratégicas en la unidad de producción CCR se ajustan a la necesidad del usuario (audiencia) para que el producto sea único. La gerencia de tecnología en CCR, usa la audiencia infantil para gestionar información a través del soporte de nuevas tecnologías (internet). La audiencia infantil es una fuente de insumos para soportar la fase de diseño del producto. Por lo tanto, en CCR el concepto de gerencia de tecnología para la producción radial se ajusta a la dimensión de la gerencia de innovación en producto, porque utiliza la tecnología mercadeo como mecanismo para satisfacer las necesidades del cliente, además integra la tecnología de información y comunicación a la gerencia del producto. Éste modo de trabajar se acerca a las características de la red para la información definida por Ait-El Hadj (1990), esta red nace de la necesidad de conciliar la cantidad de información, la dispersión de sus fuentes, y de la desviación cualitativa entre la información espontánea ofrecida y las necesidades efectivas y específicas de los solicitantes. La producción se organiza en red, porque el equipo de productores se integra con otros asociados para realizar una operación. En este caso, la operación se trata del proceso de pre-producción radial y los asociados son: la audiencia infantil, los asesores y el equipo productor CCR.

CCR se adecua al enfoque gerencial de tecnología basado en las capacidades dinámicas, planteado por Teece y Pisano, (1996) porque combina tres dimensiones básicas: estrategia de mercado, estrategia tecnológica de la firma y estrategia organizacional. El estilo gerencial de CCR se aproxima al patrón correspondiente al modelo de innovación radical de las pequeñas empresas desarrollado por Abernanthy y Uterback en 1978, porque se asocia a la acción de identificar una necesidad emergente (innovación en 
producto) o con una nueva manera de satisfacer una necesidad existente (innovación en proceso). Los mecanismos desde la empresa CCR para innovar en producto tienen relación con la actividad de gestionar información como clave para orientar la producción a la satisfacción del cliente.

Los mecanismos para la innovación en proceso se corresponden con las actividades de aprovechamiento de sinergias y alianzas para mejorar procesos productivos, consolidando conocimientos o información. Asimismo, en CCR los mecanismos para innovar en procesos están destinados a mejorar sistemáticamente las capacidades de rapidez, agilidad y calidad de las funciones de producción (innovación interna) a través del uso de nuevas tecnologías (automatización).

La página web de CCR está diseñada de tal forma, que cualquier niño del ámbito internacional, puede convertirse en una fuente de información al generar ideas y experiencias a través de la red constituida para gestionar la información y crear un sólido vínculo entre la fase de diseño y mercadeo del producto.

El servicio de información electrónica es útil para el establecimiento de estrategias de venta, lo cual contribuye directamente con el proceso de financiamiento de la estación en CCR en dos aspectos: 1) la estación promociona y vende su productos radiales, cursos y material publicitario, 2) la estación genera espacio para promocionar o vender los productos ofrecidos por sus propios anunciantes (clientes).

Quienes gerencian la tecnología en la unidad CCR también han impulsado la diversificación de la cartera de productos, mediante el ofrecimiento de cursos de producción radial infantil a todo público, eventos, información para papas, diversión.

- "RADIO TIC-TAC" (RTT): Es una unidad de producción que realiza un programa infantil en español de transmisión internacional/local, Ilamado Radio Tic-Tac. Este programa une a los niños de diferentes culturas a través de la radio. La unidad de producción RADIO TICTAC (RTT) se encuentra ubicada en territorio estadounidense. Su objetivo es que los ni ños puedan expresar sus ideas, compartir música, juegos, historias y aventuras a través de la radio. El programa se transmite cada sábado de 11:00 am a 12:00 m en 12 estaciones de Estados Unidos, a través de la cadena "Hispanic Satellite Network" (HSN), con sede en Massachusetts. Este enlace o vinculación de estaciones es una red de transmisión en cadena, que permite difundir el espacio radial en diferentes mercados, ampliando sus oportunidades de diversificación e innovación como producto. 
La unidad de producción "RADIO TIC- TAC" cuenta con una página web interactiva disponible en: http://www.tictactuk.com/tictacradio.htm y además, posee un proyecto llamado TIC (referido al uso de las tecnologías de información y comunicación) con la finalidad de realizar producciones de radio, internet y televisión con niños. El proyecto TIC permite la difusión de las producciones de RADIO TIC-TAC en países de Latinoamérica y ciudades de Estados Unidos. TIC apoya además campañas de instituciones que trabajan en favor de la infancia y promociona eventos que realizan esfuerzos para mejorar el desarrollo de niños y niñas en comunidades y países del mundo. Con esta estrategia de expansión la gerencia RTT logra ampliar la posición competitiva tanto nacional como internacional. Del mismo proyecto TIC específicamente a través del uso del sistema de internet, se extrae la información de la audiencia infantil para el diseño de las producciones de radio, a un mínimo costo.

En la fase de ingeniería del producto RTT trabaja de forma sistemática. La unidad de producción RTT recibe asesoramiento profesional por parte de un equipo de comunicadores con experiencia en el género infantil. Se gestiona la información considerando las ideas y necesidades del usuario (audiencia infantil) a través del correo electrónico, correo postal o vía telefónica.

RTT enfoca la fase de producción hacia el desarrollo de temas específicos, seleccionados por la fuente primaria de información: los niños, o hacia propuestas de temas de acción social aportados por la asesoría de profesionales.

La unidad productiva RTT tiene un modo de funcionar basada en tres dimensiones: Proyecto de producción sistemática de información; Proyecto comunicacional y Proyecto de acción social. Este tipo de funcionamiento permite a RTT aprovechar la mayor cantidad de vínculos para cubrir la información y la acción. RTT para desarrollar los proyectos de comunicación radiofónica cuenta con material y recursos propios. Además, la gerencia de RTT, aprovecha los vínculos con medios de comunicación e instituciones ligadas a la atención de la infancia para crear sinergias y conformar equipos de acción basados en la combinación capacidades.

En la unidad productora RTT la implementación de nuevas tecnologías (NT) es un soporte esencial para el proceso de realización del producto radial. Actualmente, RTT funciona aprovechando las bondades de la tecnología digital y el servicio internet para apoyar todos los procesos de diseño, producción, transmisión y mercadeo del producto radiofónico. 
Precisamente la estrategia publicitaria que soporta el proceso de mercadeo del producto en RTT se realiza a través del proyecto TIC. La imagen publicitaria de la empresa se proyecta por televisión, internet, y radio (red satelital). Estos dos últimos medios, son el soporte del proceso de distribución del producto final generado por RTT.

El desarrollo de proyectos comunicacionales TIC, apoya el mercadeo del producto en diferentes culturas y países. RTT desarrolla servicios complementarios como información electrónica de interés para los niños y, además, de la misma manera que da a conocer su producto, apoya eventos y campañas infantiles a través de los medios de difusión que utiliza: radio, televisión e internet. La unidad de producción RTT recibe financiamiento del Estado norteamericano, a través de la realización del proyecto TIC.

El estilo de gerenciar la tecnología en la unidad de producción RTT se orienta hacia la innovación en producto, básicamente, como resultado de la integración de la estrategia de mercado, la estrategia tecnológica y la estrategia organizacional, RTT también ha entrado en la dimensión de la innovación en procesos generando mejoras como: reducción de costos y aumento de la productividad de los procesos de diseño, realización, mercadeo y venta del producto.

La unidad de producción RRT responde a las características de las empresas que emplean redes de acción. Estas redes según Ait El-Hadj (1990) puede tener múltiples formas, sobretodo si se incluye el desarrollo internacional y la acción comercial. El autor también señala que el desarrollo de este tipo de red se apoya al menos en dos atributos de la industria contemporánea: $1^{\circ}$ la complejidad tecnológica de la actividad industrial y, particularmente, la dispersión de los conocimientos de las tecnologías que van apareciendo, obligan a recurrir a numerosas empresas para poder realizar nuevos objetos técnicos, y $2^{\circ}$ el paso de una concepción de la producción en términos de integración física, a una concepción en términos de coherencia de las decisiones, de integración de la información y la satisfacción del cliente (audiencia).

- “RADIO LOLLIPOP"(RL): se originó en el año 1979, en el Hospital Infantil Queen Mary's Hospital for Children de Inglaterra. RL es una unidad de producción de programas radiales infantiles, basados en actividades lúdicas y enseñanzas orientadas por voluntarios, con el fin de ayudar a los pequeños pacientes a desarrollarse, mediante una terapia sincronizada, con emisiones radiales transmitidas en circuito cerrado.

En agosto de 1996 el Miami Children's Hospital se convirtió en el primer hospital en los Estados Unidos en ofrecer RL a sus pacientes. El diseño del contenido del producto esta a 
cargo de un equipo multidisciplinario de profesionales que se encarga del proceso de asesoramiento en esta etapa de la producción. Investigaciones han comprobado que las actividades lúdicas tienen un componente esencial para el desarrollo del niño; este conocimiento de base científica sumado a los resultados de la aplicación de la técnica lúdica fusionada con la tecnología radiofónica, constituye la combinación perfecta de conocimientos para implementar el proyecto RL en hospitales para la atención de niños.

La idea de crear RL se originó de la siguiente tesis: “... los niños hospitalizados deben recibir el necesario aliento y apoyo para participar en juegos y actividades de aprendizaje", disponible en: http://www.mch.com/patient-sp/radio_lollipop. Desde esta perspectiva de acción, RL ha tenido un enorme éxito. En la actualidad existen unidades de producción RL en más de 15 hospitales en Inglaterra, Australia, Nueva Zelanda y los Estados Unidos, donde, cada año, más de un cuarto de millón de niños disfrutan el servicio de Radio Lollipop.

Cada unidad de producción Lollipop, es una estación de radio totalmente equipada, conformada por un grupo de especialistas en comunicación radiofónica, médicos y voluntarios debidamente entrenados, para involucrar a los pacientes en las actividades lúdicas a través de programas radiales con enfoque de acción social. Este enfoque orienta la realización del producto. No importa la edad del niño y tampoco la temática. Lo importante es estimular el progreso del ni ño considerando su necesidad como usuario a través de la combinación de la técnica radiofónica y el juego.

Para efectos de este trabajo se considerará como objeto de análisis la unidad de producción RL perteneciente al Miami Children's Hospital. La unidad de producción RL del Miami Children's Hospital funciona todos los martes, miércoles y jueves de 6:30 a 8:30 pm en el tercer piso del hospital contiguo al salón de juegos.

La unidad de producción RL ofrece una programación de entretenimiento exclusiva para los pacientes. Su política es formar voluntarios; personas de 18 años en adelante que puedan dedicar dos horas a la semana trabajando y jugando con niños. En la fase de diseño del producto RL es un caso muy particular, porque obligatoriamente, algún miembro del equipo multidisciplinario que imparte la asesoría para el diseño del programa, debe dominar el conocimiento científico especializado en referencia a la atención de los niños (pacientes). Por tal razón la gerencia RL desempeña un proceso de selección de sus fuentes de información para la configuración de la idea del producto. Se hace énfasis en los profesionales ligados a la atención de los niños de los hospitales, como: psicopedagogos, psicólogos infantil, educadores especiales y pediatras entre otros. La 
gestión de la información se hace a través de la línea telefónica: Referidos médicos.1888-MCH-DOCS.

En el proceso de realización del producto los pacientes son quienes presentan los programas. En este caso los niños por tomar el lugar de locutores del programa pasan a formar parte del equipo de realizadores.

El grupo de voluntarios RL además de llevar a cabo el programa, tienen la función de visitar a los pacientes en sus respectivas habitaciones, para leerles cuentos y alentarlos a participar en los programas y a ganar premios. La distribución de estas funciones así como las tareas de realización del producto son asignadas al grupo de voluntarios según sus habilidades personales. La dedicación de estos voluntarios a RL mereció el prestigioso premio American Hospital Association Award for Volunteer Exellence, entregado en una ceremonia en Washington, DC.

Los realizadores del proceso de producción de RL se organizan por objetivos, en equipos de trabajo multidisciplinarios y en grupos voluntarios integrando capacidades: conocimiento radiofónico, médico, y de la experiencia del grupo de voluntarios que atienden a los pacientes.

En relación al proceso de mercadeo de producto en $\mathrm{RL}$ es necesario hacer cuatro observaciones importantes: 1) la estrategia publicitaria está soportada en el uso de los medios de difusión radio e internet; 2) RL cuenta con una Página Web, sólo con propósitos informativos y promocionales para aumentar el número de voluntarios y el patrocinio; 3) La distribución del producto se realiza desde los centros de atención médica (hospitales), 4) La imagen de RL se difunde a través de internet promocionando reuniones de capacitación para grupos y comunidades voluntarias en todo el mundo. El ofrecimiento gratuito de ésta capacitación, para producir RL es un servicio complementario y una estrategia de la gerencia para apoyar el mercadeo del producto. La gerencia de RL se encarga de dictar exclusivamente el entrenamiento de los voluntarios con el propósito ampliar su cartera de patrocinantes en todo el mundo.

La unidad de producción RL recibe donaciones de individuos y empresas; sin embargo, también cuenta con el patrocinio de grandes empresas, tanto a nivel local como internacional. La visión de la gerencia de RL es fundar más unidades de producción RL en hospitales orientados a la atención de niños en todos los países del mundo.

La gerencia de tecnología en RL se apoya en la combinación dinámica de capacidades 
internas y externas a la organización. Para este proceso la gerencia atrae a su unidad productiva voluntarios a quienes entrena y de los cuales también aprovecha la experiencia y conocimiento para el trabajo con los niños. A tales voluntarios no se les remunera. En compensación por su trabajo se les premia y se les brinda la oportunidad de desarrollar su espíritu altruista. Estos voluntarios son ubicados en equipos de trabajo.

El estilo de gerenciar la tecnología en RL se orienta hacia la innovación en producto, adaptando el proceso productivo en sus distintas fases a la necesidad de los usuarios (niños pacientes).

La unidad productiva RL también se corresponde con el patrón de innovación en proceso, ya que emplea nuevas tecnologías para soportar los procesos de mercadeo (promoción y financiamiento de su producto) y la estrategia organizacional de expansión que consiste en crear puntos focales de responsabilidad en diferentes hospitales del mundo, desarrollando equipos de trabajo Lollipop.

- "La Chacamota" (Ch): Según Vázquez (1999) productora-realizadora de la empresa de producción radial "LA CHACAMOTA" afirma que ésta es una empresa independiente que funciona como una unidad de producción para el gobierno de Colima- México, realizando el programa de radio infantil: “LA CUEVA DEL CUENTERO" diseñado y vendido en series cortas (micros) para que sean transmitidas en las escuelas con el propósito de complementar el proceso de la enseñanza de la lecto-escritura en los niños.

La unidad de producción LCH, posee una modalidad especial de financiamiento: Becas de creación y ventas de servicio. La idea de crear el colectivo 4 LCH consistió en el aprovechamiento de las habilidades personales de todos sus miembros. Además, cada miembro de la organización posee conocimientos sobre producción radiofónica; y formación profesional de base: "Todos somos comunicadores sociales".

La misión de "LA CHACAMOTA" ( $\mathrm{LCH}$ ) como unidad de producción radiofónica dirigida a la infancia consiste en gestionar proyectos viables para la producción independiente, y la creación de productos audiovisuales, de manera didáctica y divertida, con el fin de informar y formar al espectador. Los principales valores de la organización son: $1^{\circ}$ tener gente muy creativa y $2^{\circ}$ darse a conocer en su mercado a través de premios y méritos propios.

"LA CHACAMOTA" (LCH) es una organización que se constituyó inicialmente como asociación civil y actualmente es productora independiente. Como productora comenzó a 
funcionar desde 1994. Está constituida por un equipo de emprendedores, que trabajan en la ciudad de Colima-México. La unidad de producción LCH pertenece a la clasificación de pequeña empresa por su bajo número de empleados; es una productora que no tiene competidores en el mercado porque en la ciudad de Colima nadie se arriesga a tratar los temas que LCH produce, ni a hacer radio independiente (Vázquez, 1999).

La combinatoria de capacidades en la unidad productiva LCH es posible gracias a la configuración de la organización en equipos y al establecimiento de vínculos con proveedores de tecnologías. Los procesos organizacionales en LCH permiten combinar capacidades internas e impulsar la evolución de los productos en múltiples series cortas de radio.

En cuanto a la gerencia de tecnología una de las fortalezas más evidentes del colectivo LCH según Vásquez (1999) es la experiencia de su equipo de trabajo en referencia a la producción radiofónica infantil. Los miembros de la organización tienen conocimientos sobre radio, audio, nuevas tecnologías, televisión y un poco de cine. Además, se caracterizan por ser muy creativos en materia de proyectos educativos. El equipo de trabajo conoce empresas más grandes de producción radial que le ayudan o facilitan material, equipos y, además, satisfacen cualquier necesidad de realización. Según Vázquez (1999), LCH es la única empresa local en su género, que funciona a través de vínculos para combinar competencias y medios complementarios.

La unidad productiva LCH, está compuesta por cinco miembros de planta, uno de sus componentes asume el punto focal de responsabilidad, al coordinar cada nuevo proyecto, y es el enlace con la institución contratante. La empresa no tiene organigrama establecido; la organización desarrolla actividades mediante una estructura horizontal, orientada por los lineamientos donde intervienen una serie de variables internas de la organización, como la rotación e integración de funciones entre sus miembros. La organización del trabajo dentro del colectivo se hace según las áreas fuertes del conocimiento. Sus miembros pueden rotar funciones en algunos proyectos, según su nivel de experiencia y dominio de competencias.

LA CHACAMOTA (LCH) no tiene director establecido posee un Íder (Rabí Hernández), quien tiene a su cargo la gerencia de los proyectos. Él es el guionista principal, encargado del diseño por computadora, compone canciones y canta. Los miembros de esta organización son multidisciplinarios y multifuncionales.

LA CHACAMOTA como organización también ha logrado una mayor integración de la 
producción a los procesos de la gerencia del producto (diseño, realización, mercadeo etc.). Así, la licenciada Karina Robles; quien administra el dinero de la empresa, a su vez produce, elabora guiones y hace voces. El licenciado Sergio Velasco que es el encargado de la edición por computadora y el músico, también participa en la realización de las imágenes promocionales. Roberto Levy, comunicador social, tiene a su cargo la edición analógica y a su vez hace voces en los programas de radio. Marina Vázquez, es magíster en comunicación e industrias audiovisuales; se encarga de producir (hacer voces, entrevistas, guiones), y específicamente, se encarga del diseño creativo y de contratar recursos humanos o talentos. Según Vázquez a LCH le falta el contador y el vendedor, pero cuando lo necesitan lo contratan.

El uso de nuevas tecnologías ha generado tanto influencias positivas como negativas a la unidad de producción LCH “...la edición por computadora nos ha ayudado a mejorar la calidad, aunque nos tardamos muchas horas en editar un sólo programa, el poder quemar en disco compacto da mejor presentación así como el diseñar e imprimir la portada de los trabajos" (Vázquez, 1999).

Para asimilar este conocimiento y generar capacidades internas en cuanto al manejo de nuevas tecnologías, la gerencia de la unidad LCH ha aprobado la formación, y constante actualización de su personal. Cuestión que la organización LCH considera vital para crear competitividad.

En el estilo de gerencia de tecnología en LCH se observan los siguientes procesos: 1) la fase de diseño de producto es apoyada por el asesoramiento profesional de comunicadores con experiencia en el género infantil y por el conocimiento que aportan empresas afines; la información para la configuración del producto proviene del aporte de la audiencia infantil y los profesionales ligados a la infancia, quienes han sido seleccionados como principales fuentes informativas. La gestión de la información se realiza a través de e-mail (correo electrónico), correspondencia (servicio postal) y mediante la investigación period ística de los miembros de LCH; 2) en la fase de realización del producto, la las tareas se realizan en equipo para aprovechar habilidades personales, conocimientos profesionales y dominio en la aplicación de NT; 3) en la fase de mercadeo de producto la estrategia consiste en aprovechar los bajos costos del servicio postal mexicano, para distribuir y a la vez mercadear el producto.

LCH promociona la serie radiofónica "LA CUEVA DEL CUENTERO" a través de diferentes estaciones de radio, en las escuelas de Colima, México, en eventos y concursos culturales de carácter internacional. “ LA CUEVA DEL CUENTERO” en el año 1998 ganó premio 
cultural internacional en Chile, por promover la enseñanza de la lecto-escritura en la población infantil de Colima-México.

La gerencia de tecnología en la unidad productiva LCH, orienta su estrategia tanto a la innovación en producto como a la gerencia del proceso, mediante un enfoque que combina ideas racionalistas con ideas soportadas en el modelo de las capacidades dinámicas y el funcionamiento del modelo de Pórter "SWOT". La gerencia de tecnología en LCH analiza las fortalezas y las debilidades de la unidad productiva a la luz de las oportunidades y amenazas externas, ayudando a los miembros de su empresa a estar conscientes de las tendencias que existen en el ambiente competitivo; para crear vínculos con otras organizaciones y aprovechar sinergias. Esto evidencia un proceso continuo de combinación de capacidades internas de la firma con capacidades externas a ella.

En la unidad de producción LCH la implementación de tecnologías digitales ha generado las siguientes implicaciones dentro del proceso productivo: 1) La automatización ha aumentado la calidad del producto y a su vez los niveles de complejidad de las tareas, exigiendo al operador un nivel de conocimiento superior. 2) Como resultado de lo anterior, aumenta el tiempo del proceso de producción por falta de capacidad operativa en el manejo del software. 3) Para superar la debilidades al respecto, la gerencia de la unidad de producción LCH ha dispuesto la participación de sus miembros en cursos de especialización cuyos contenidos se refieran al manejo operativo de la tecnología digital con el fin de actualizar los conocimientos de sus aperadores y aumentar la velocidad de sus procesos productivos.

\section{Conclusiones}

Las características más significativas del estilo de gerencia de tecnología en las UIPRI se evidencian en las siguientes estrategias: combinar capacidades tecnológicas internas y externas, implementar nuevas tecnologías, diversificar la cartera de productos, gestionar información y recursos y transmitir y vender el servicio a través del internet, gestionar redes de información y acción. Tales estrategias están orientadas sistemáticamente a desarrollar competitividad, innovar en producto, satisfacer al cliente (audiencia), mantener la eficiencia, planificar y organizar actividades productivas de acuerdo a las oportunidades ofrecidas en el entorno. Tal estilo se basa en el enfoque de las capacidades dinámicas de la firma, es decir, en la integración de las estrategias de mercado, tecnológica y organizacional (Teece y Pisano, 1994).

El estilo de gerencia de tecnología en las UIPRI se aproxima al segundo patrón del 
modelo planteado por Abernathy y Utterback 1978 (Añez, 1995) asociado a la identificación de una necesidad emergente (innovación en producto) y con una nueva manera de satisfacer una necesidad existente (innovación en proceso). La necesidad emergente fluye de los requerimientos de la audiencia (niños) y el producto se materializa a través del enlace entre las fases de diseño y mercadeo. La identificación de una nueva manera de satisfacer una necesidad existente se refiere a la aplicación de nuevas tecnologías; incorporación del internet, software y otros productos tecnológicos para mejorar los procesos de producción -y también la transmisión- radiofónica.

El estilo también es coherente con el enfoque de gestión estratégica y operativa de la tecnología planteado por Ait-El-Hadj (1990) para responder sistemáticamente a la innovación de la siguiente forma: $1^{\circ}$ Para innovar en producto ( innovación externa) se emplean mecanismos de gestión de información (redes y vigilia) para aprovechar capacidades, sinergias y vínculos. $2^{\circ}$ Para innovar en proceso (innovación interna) se incorporan NT, mecanismos para automatizar, es decir mejorar capacidades de rapidez, agilidad y de calidad de las funciones de producción.

Este estilo también se articula con el nuevo concepto de producción radial planteado por Bethencourt (1998), el cual se ajusta a la gerencia de la innovación en producto; incorporando la tecnología mercadeo como mecanismo para satisfacer las necesidades del consumidor (cliente-audiencia). Además, integra las tecnologías de información y comunicación a la gerencia del producto.

\section{Notas}

1. Alvin Toffler (citado por Jeremy y Tony Hope: 2000:12), describe tres periodos de evolución económica, la ola agrícola, que duró desde 8000 A.C., hasta mediados del siglo XVIII; la ola industrial, que duró hasta avanzado el siglo XX; y la Ola de la información, que empezó en los años sesenta y continuará vigente durante varias décadas. Por supuesto, estas fechas son aproximadas y se solapan. La primera ola fue impulsada por el trabajo físico, la segunda ola por las máquinas y los trabajadores manuales, y la tercera ola por la tecnología de la información y los trabajadores con conocimiento.

2. Para efectos de este trabajo se tomará empresa y organización como sinónimos, se hará referencia a ambos indistintamente, con independencia de que tengan una misión comercial o actúen sin fines de lucro.

3. En una intervención pronunciada en el Taller de Expertos sobre Política Industrial, efectuado del 16 al 17 de septiembre de 1996, en la sede del SELA, en Caracas Venezuela. 
4. La palabra "colectivo" usada por Vázquez, debe entenderse como sinónimo de equipo de trabajo.

\section{Referencias Bibliográficas}

1. Abemathy, W.J. Utterback, J.M. (1978), "Patterns of Industrial Innovation", Tecnology Review, 80, june/july. Inglaterra.

2. Ait-El-Hadj, Smail (1990), Gestión de la tecnología: la empresa ante la mutación tecnológica. Ediciones Gestión 3000. Barcelona, España.

3. Alonso, Juan (2001), Diccionario Enciclopédico ESPASA. ESPASA CALPE S.A., España.

4. Añez, Celina (1995), The I nnovation Theory. A summary of its Evolution, Literature review, Dphil project. University of Brighton, UK.

5. Avalos, Ignacio (1994), “Transferencia de tecnología”, en E. Martínez (ed) Ciencia, tecnología y desarrollo, CEPAL-ILPES/UNESCO/UNU/CYTED-D, Caracas: Nueva Sociedad, 411-454.

6. Barreto, Alfonso (2000), Introducción a la Planificación y la Gerencia de Ciencia y Tecnología. Apuntes de clase. Profesor contratado del Programa de Maestría en Planificación y Gerencia de Ciencia y Tecnología dictado por la División de Estudios para Graduados de la Facultad Experimental de Ciencias de la Universidad del Zulia. Maracaibo, Venezuela.

7. Bethencourt, Tomás (1998), Nueva concepción de la producción radiofónica. Entrevista Personal. Profesor y Coordinador Académico de la III Maestría en Comunicación e Industrias Audiovisuales en el espacio Iberoamericano de La Universidad Internacional de Andalucía. Sede Iberoamericana Santa María de La Rábida. Abril Huelva. España.

8. Colorín Colorradio Emisora Infantil Pre Juvenil de Colombia. Disponible http://www. colorincolorradio.com/quees.asp (Febrero 10, 2001).

9. Hope Jeremy y Hope Tony (2000), “Competir en la tercera Ola”. Los diez temas clave de la dirección en la era de la información. Un libro de Harvard Bussiness School Press. Gestión 2000 p.12, 2da. Edición. Barcelona, España. 
10. “Gus” Gaynor, Gerard (1999), “Gestión tecnológica, descripción, campo de acción e implicaciones". En: Gus Gaynor, G. (comp.), Manual de gestión en tecnología, una estrategia para la competitividad de las empresas, Tomo 1. ( pp. 3-31). Mc-GrawHill. Santa Fe, Bogotá, Colombia.

11. Machado, Fernando (1996), La política industrial y la política tecnológica: los retos actuales. Capítulo del SELA, 48, octubre-diciembre, Venezuela.

12. Martínez, Miguel (1996), Comportamiento humano: nuevos métodos de investigación. Trillas. M éxico.

13. Radio Tic-Tac Disponible en http://www.tictactuk.com/tictaradio.htm (febrero 10, 2001).

14. Rojas, Luis (1994), “El gerente: paradigmas y retos para su formación”. Encuentro Educacional, 1, 01-28.

15. Servicio a Pacientes Radio Lollipop. Disponible: http://www.mch.com/patient-sp/ radio_lollipop.htm (Febrero 10, 2001).

16. Teece David and Pisano, Gary (1994), "The dynamic capabilities of firms: an introduction", I ndustrial and Corporate Change 3(3): 537-556.

17. Tidd Joe, Bessant John \& Pavitt, Keith (1997), “Managing Innovation. Integrating Technological, Market and Organizational Change". Chichester: John Wiley \& Sons.

18. Vásquez, Marina (1999), "Empresa de Producción Radial La Chacamota". Cuestionario Postal. Productora y Realizadora de radio infantil. Comunicadora Social. Master en Comunicación e Industrias Audiovisuales. Colima, México. 